\title{
COMPARATIVE STATISTICAL ANALYSIS OF SELECTED CONTROL CHARTS FOR HIGHLY CAPABLE PROCESSES
}

\author{
Eva Jarošová, Darja Noskievičová
}

\section{Introduction}

The statistical process control (SPC) is widely used in industry. Its aim is to achieve process stability and improve capability through the reduction of variability and it is included for example in the Six Sigma or Lean Six Sigma methodologies. The control of attribute data represents a considerable part of it. Until recently, the same approach was used to monitor variables or attributes data. In this approach, subgroups of items are taken from a process and sample characteristics are plotted in a control chart to see whether their variation is only random or whether it is affected by an assignable cause. The presence of such cause is indicated by exceeding the control limits that are based on the sample characteristic distribution.

When items are classified as conforming or nonconforming, the proportion or the count of nonconforming units in a process (fraction nonconforming) is traditionally monitored by a $p$ chart or np chart. Due to new manufacturing technologies and concepts, many processes are of such a high quality that the fraction nonconforming or the probability of observing a nonconforming unit is very small. This probability is a parameter of the background binomial distribution and the size of the subgroups would have to be enormously large to enable the normal approximation, on which the computation of control limits is based.

The impossibility to accomplish such conditions has the following consequences: real properties of a chart such as the average run length (ARL) or the risk of false signal $\alpha$ differ from those assuming a normal distribution and the lower control limit of a $p$ chart is located at zero and does not enable the recognition of the process improvement. Moreover, the appearance of a $p$ chart containing most zero points is inappropriate. Consequently, the concept based on the sample characteristics is no longer useful for high-quality processes.

Several alternatives based on the Bernoulli process rather than on the normal approximation have been recently presented. These methods assume continuous inspection. But not necessarily $100 \%$ items must be inspected. For example Reynolds and Stoumbos (1999) admit a situation when "the production rate is higher than the inspection rate", Szarka and Woodall (2011) also mention an interval sampling when the items are inspected at scheduled periods. Bourke (2001) emphasizes that "the pattern of the sampling inspection can be quite haphazard without causing any difficulty" for the performance of the control chart he had studied.

In the Bernoulli process, random values $x_{i}=0$ or $x_{i}=1, i=1,2, \ldots$, express whether an inspected item is conforming or nonconforming, respectively. The in-control state of a process is defined by a constant probability $p_{0}$ of an occurrence of a nonconforming item. Usually a sustained shift to the out-of-control state is considered; the process repetitively produces units at level $p_{0}$ until it suddenly shifts to an unacceptable level $p$ and remains at this level until a remedial measure is taken. Especially the situation $p>p_{0}$ must be detected as soon as possible and sometimes a minimum unacceptable value for $p$ is given. Sometimes the inverse inequality $p<p_{0}$ may be of interest, too, because it indicates a process improvement.

In the new approach, the number of units $Y_{i}$ being inspected until the $r$-th nonconforming one has been observed is followed, where $r \geq 1$. Either the geometric (for $r=1$ ) or the more general negative binomial distribution of $Y_{i}$ are assumed. Apart from the Shewhart-type charts based on these two distributions (often called CCC or CCC-r charts) the CUSUM or EWMA charts can be constructed. 
The control limits of the CCC and CCC-r charts are the probability limits; they are constructed directly as percentiles for a given risk of false signal $\alpha$.

Cumulative sum (CUSUM) charts use information from all the prior observations (in this case the run lengths $Y i$ defined above) and they are considered to be an efficient alternative to the Shewhart chart when small process shifts are of interest. Usually $r=1$ is considered and the corresponding CUSUM chart is called a geometric (or CCC) CUSUM.

Exponentially weighted moving average (EWMA) charts are also based on all the prior observations and have a similar efficiency as the CUSUM charts.

Numerous authors studied efficiency of these control charts (see further) but only few case studies have been published, see (Chang \& Gan, 2001) or (Di Bucchianico et al., 2005)

In this paper the CCC-r charts and the geometric CUSUM chart are analyzed. The aim of the paper is to compare the CCC-r and geometric CUSUM charts by their speed of detecting an upward shift in the fraction nonconforming when they are applied to a process with the target fraction nonconforming of the order of $100 \mathrm{ppm}$. Both types of charts are then applied to a real process of the electronic assembly that has become a basis for the study. To our best knowledge no study dealing with such a small fraction nonconforming together with the low risk of false alarm has been published yet.

\section{Control Charts Description}

For our comparative analysis we have chosen three types of the control charts based on the Bernoulli process: the CCC, CCC-r and CCC-CUSUM charts. Their theoretical basis will be described in this section.

\subsection{Chart}

The CCC chart was designed by Calvin (1983) and further studied and expanded by Goh (1987), Bourke (1991) or Xie et al. (1995). The name CCC stands for the cumulative count of conforming units, but more frequently, the variable $Y$ that is monitored includes also the immediately following nonconforming unit. Other names such as the conforming run length $\mathrm{CRL}$ in $(\mathrm{Wu}, 2000)$ or run-length $\mathrm{RL}_{1}$ in (Bourke, 1991) can be met in literature. We consider the latter case but we will use the inaccurate but more known name CCC in our paper.

Variable $Y$ follows the geometric distribution $\mathrm{G}(p)$ with the probability function

$$
f(y)=p(1-p)^{y-1}, y=1,2, \ldots,
$$

where $p$ is the probability of observing a nonconforming unit in any inspection. The centreline of the CCC chart corresponds to the median of the geometric distribution

$$
C L=\frac{\ln \frac{1}{2}}{\ln (1-p)},
$$

and the two-sided probability control limits (the upper control limit $U C L$ and the lower control limit $L C L$ ) are based on the chosen risk of false alarm $\alpha$ (Xie et al., 2002):

$$
U C L=\frac{\ln \frac{\alpha}{2}}{\ln (1-p)}, L C L=\frac{\ln \left(1-\frac{\alpha}{2}\right)}{\ln (1-p)}
$$

As these limits are highly asymmetric, a logscale is sometimes used (see for instance Xie et al., 2002).

As soon as a nonconforming unit is observed, the value $Y i$ is plotted on the chart and counting starts again from zero. The interpretation of this control chart is quite different from the interpretation of the conventional Shewhart p chart: the point above UCL indicates the probable process improvement while the point below $L C L$ shows the probable process deterioration.

When only an upward shift in the process fraction nonconforming is of interest, the onesided lower control limit is given by

$$
L C L=\frac{\ln (1-\alpha)}{\ln (1-p)} .
$$

For the conventional Shewhart or CUSUM charts, when both the sample sizes and the sampling intervals are equal, the average run length $A R L$ can be used to assess the efficiency of a chart. It represents the expected number of samples before the first signal occurs. This measure is inappropriate with charts based on the Bernoulli process. The observations $Y_{i}$ 
correspond to different numbers of inspected units and this fact must be taken into consideration. That is why another measure has been introduced. Reynolds and Stoumbos (1999) use the average number of observations to signal ANOS that represents the expected number of inspected items to the signal. The average time to signal ATS, the average number inspected $A N I$, the average number of items sampled ANIS are different names for the same measure used in Wu et al. (2000), Bourke (1991) and Chang and Gan (2001), respectively. In our paper we use the acronym ANOS, or $\mathrm{ANOS}_{0}$ for the in-control process with fraction nonconforming $p_{0}$.

\subsection{CCC-r Chart}

To improve the sensitivity of detecting small upward shifts in the fraction nonconforming, the chart based on $r$ successive run lengths has been developed. Bourke (1991) first considered $\mathrm{RL}_{2}$ chart for $r=2$ with the moving sums $R L_{2}=Y_{i-1}+Y_{i}$ for $i=2,3, \ldots$, but later the separated sums $Y_{1}+Y_{2}, Y_{3}+Y_{4}$ etc. were used, see e.g. (Wu et al., 2001). More general case $r \geq 2$ is considered in Xie et al. (1999), Kaminsky et al. (1992), Xie and Goh (1997). Often $r$ values of 2 or 3 are recommended, see Wu et al. (2000) or Schwertman (2005). Ohta et al. (2001) constructed an economic model to find the best value of $r$. All these charts are based on a negative binomial distribution of the monitored variable. Common name for these charts is the CCC-r chart, although other names may appear, e.g. SCRL chart in Wu et al. (2000).

The cumulative count of units until the r-th observed nonconforming unit follows a negative binomial distribution with the probability function

$$
\begin{aligned}
& f(y)=\left(\begin{array}{c}
y-1 \\
r-1
\end{array}\right) p^{r}(1-p)^{y-r}, \\
& y=r, r+1, r+2, \ldots
\end{aligned}
$$

Assuming that the probability of a nonconforming unit is $p$, the two-sided control limits and the centerline are determined by solving the following equations (see Chen \& Cheng, 2010):

$$
F(U C L)=\sum_{y=r}^{U C L}\left(\begin{array}{l}
y-1 \\
r-1
\end{array}\right) p^{r}(1-p)^{y-r}=1-\frac{\alpha}{2},
$$

$$
\begin{aligned}
& F(L C L)=\sum_{y=r}^{L C L}\left(\begin{array}{l}
y-1 \\
r-1
\end{array}\right) p^{r}(1-p)^{y-r}=\frac{\alpha}{2}, \\
& F(C L)=\sum_{y=r}^{C L}\left(\begin{array}{l}
y-1 \\
r-1
\end{array}\right) p^{r}(1-p)^{y-r}=\frac{1}{2} .
\end{aligned}
$$

When only an upward shift is followed, the one-sided lower control limit must satisfy the equation

$$
F(L C L)=\sum_{y=r}^{L C L}\left(\begin{array}{c}
y-1 \\
r-1
\end{array}\right) p^{r}(1-p)^{y-r}=\alpha
$$

for the specified risk of false signal $\alpha$. The interpretation of the CCC-r chart is similar to the CCC chart interpretation.

With an increasing value of parameter $r$ the CCC-r chart is more sensitive to small upward shifts in $p$. But on the other hand more and more Bernoulli observations are needed to obtain a point in the chart and therefore the inspection cost increase (see e.g. Ohta \& Rahim, 2001). For that reason it is necessary to set the optimal parameter $r$.

\subsection{Geometric CUSUM Chart}

The geometric CUSUM chart was proposed by Bourke (1991). It is based on the schemes introduced in (Page, 1954) that can be expressed by

$$
\begin{aligned}
& S_{i}^{+}=\max \left(0, S_{i-1}^{+}+Y_{i}-K^{+}\right), \\
& S_{i}^{-}=\min \left(0, S_{i-1}^{-}+Y_{i}-K^{-}\right), i=1,2, \ldots
\end{aligned}
$$

These schemes can be used to detect downward or upward shifts in a distribution of $Y_{i}$. If variables $Y_{i}$ follow a geometric distribution and the process deteriorates, i.e. if the fraction nonconforming $p$ increases, values of $Y_{i}$ less than $K^{-}$predominate and $S_{i}^{-}$becomes more and more negative. As soon as $S_{i}^{-}$drops under the specified limit $H^{-}$for some $i$, an out-of-control signal is given. The value of $\mathrm{H}^{-}$determines the chart performance, i.e. ANOS $_{0}$ and ANOS. The more negative it is, the longer the time to signal.

To avoid the negative values of $S_{i}^{-}$, the lower Page scheme is often presented in the form $S_{i}^{-}=\max \left(0, S_{i-1}^{-}-Y_{i}+K^{-}\right)$. Here the original expression given by (10) is used, with an omitted minus sign in the upper index. 
Values of the CUSUM statistic are then zero or negative and the lower limit $H$ is negative.

When a shift from in-control $p_{0}$ to a higher value $p$ is to be detected, the minimum value $p_{1}$ that is considered to be inappropriate must be chosen. The level of the fraction nonconforming is acceptable for $p \leq p_{0}$, and unacceptable for $p \geq p_{1}$. As a matter of fact, hypotheses $\mathrm{H}_{0}: p=p_{0}$ versus $H_{1}: p=p_{1}$ are tested. Using the Wald sequential probability test and the geometric distribution given by (1), the reference value $K$ is

$$
K=\frac{\ln \frac{p_{1}\left(1-p_{0}\right)}{p_{0}\left(1-p_{1}\right)}}{\ln \frac{1-p_{0}}{1-p_{1}}} .
$$

Frequently $S_{0}=0$ is used but a head-start can be chosen to detect an initial out-of-control state quickly (see Bourke, 1991).

The determination of the lower limit $H$ is somewhat complicated. When the Markov chain approach is used, the number of $H$ transient states is quite large and computational problems arise. Bourke (1991) suggests approximating the geometric distribution by an exponential distribution, grouping states into a substantially less number of categories and then using an approach for a continuous variable based on transitions among the categories by Brook and Evans (1972). Reynolds and Stoumbos (1999) introduced the corrected diffusion approximation and presented formulas for the Bernoulli CUSUM chart, which is closely related to the geometric CUSUM chart:

$$
\operatorname{ANOS}\left(p_{0}\right) \approx \frac{\exp \left(H_{B}^{*} r_{2}\right)-H_{B}^{*} r_{2}-1}{\left|r_{2} p_{0}-r_{1}\right|}
$$

where

$$
\begin{gathered}
r_{1}=-\ln \left(\frac{1-p_{1}}{1-p_{0}}\right) \quad r_{2}=\ln \left(\frac{p_{1}\left(1-p_{0}\right)}{p_{0}\left(1-p_{1}\right)}\right), \\
H_{B}^{*}=H_{B}+\varepsilon\left(p_{0}\right) \sqrt{p_{0}\left(1-p_{0}\right)},
\end{gathered}
$$

$$
\varepsilon\left(p_{0}\right) \approx\left\{\begin{array}{c}
0.410-0.0842 \ln p_{0}-0.0391\left(\ln p_{0}\right)^{3}-0.00376\left(\ln p_{0}\right)^{4}-0.000008\left(\ln p_{0}\right)^{7} \\
\text { if } 0.01 \leq p_{0} \leq 0.5 \\
\left(\sqrt{\left(1-p_{0}\right) / p_{0}}-\sqrt{p_{0} /\left(1-p_{0}\right)}\right) / 3 \\
\text { if } 0<p_{0}<0.01
\end{array}\right.
$$

$H_{B}^{*}$ can be obtained from (12) by means of the Excel function Goal Seek.

Once the parameter $H_{B}$ of the Bernoulli CUSUM chart has been found, the parameter $H_{G}$ of the geometric CUSUM chart can be expressed as (Szarka \& Woodall, 2012):

$$
H_{G}=-m\left(H_{B}-1\right) \text {. }
$$

In this paper, the lower limit $H$ was set at first approximately using the approach by Reynolds and Stoumbos (1999), and then adjusted by means of simulations:

1. For a specified value of $A N O S_{0}$, an approximate value of the limit $H_{B}$ of the Bernoulli CUSUM that is implicitly included in the formula (12) was calculated and the corresponding value of the limit $H_{G}$ of the geometric CUSUM was determined from (13).
2. The value of $H_{G}$ was refined iteratively through simulations so that the resulting ANOS $_{0}$ is as close to the specified value as possible. It means that run lengths $Y_{i}$ for $i=1,2, \ldots$, from $\mathrm{G}\left(p_{0}\right)$ were generated and the CUSUM characteristic $S_{i}$ was calculated as well as the cumulative sum of run lengths (or the number of Bernoulli observations) NOS $_{i}$. As soon as $S_{i}$ decreased under the lower limit $H$ for some $i=l$, the simulation was interrupted and NOS, was stored. This cycle was repeated 10,000 times, i.e. ANOS $_{0}$ was estimated as an average from 10,000 NOS values. Then the value of $H_{G}$ was modified and another 10,000 cycles were performed. This procedure was repeated until the nearest possible value to the theoretical $\mathrm{ANOS}_{0}$ was attained. The final value $H$ of the parameter obtained 
by this iterative way was used in the subsequent simulations.

\section{Aspects of the Control Chart Performance Assessment}

When the performance of different types of control charts is to be compared, a suitable criterion must be chosen. It is common to compare the efficiency of charts that have roughly equal $\mathrm{ANOS}_{0}$ (see e.g. Reynolds \& Stoumbos, 1999; Wu et al., 2000).

In the Shewhart-type charts, the probability of a signal at the moment when a nonconforming unit has been observed depends only on the length of the current run, however, the value of the CUSUM statistic is affected by the past history of the process. Therefore the zero-state and steady-state analysis is distinguished at the CUSUM chart if ANOS is examined, see e.g. (Szarka \& Woodall, 2011; 2012). In the zero-state analysis a shift is supposed to be present when the process monitoring begins. The steady-state analysis is based on the assumption that a large number of conforming units were inspected before a shift occurred.

In addition, the moment of the process shift (the change point) affects ANOS in charts based on the conforming counts. The steadystate fixed-shift model assumes that the shift occurs immediately after a nonconforming item is found. Wu and Spedding (1999) were the first to point out the problem in the CCC chart and considered the alternative steady-state randomshift model, when a shift may occur at any time during monitoring. The time to the change point is assumed to follow an exponential distribution (Wu et al., 2000) or a uniform distribution (Stoumbos \& Reynolds, 1997). The random shift model is further considered in $\mathrm{Wu}$ et al. (2001) or Bourke (2006).

A number of comparative studies were performed. In Bourke (1991) the $\mathrm{RL}_{2}$ chart and the geometric CUSUM were compared and the zero-state ANOS was determined. The in-control fraction nonconforming $p_{0}$ was 0.01 and the ANOS curves showing dependence on the fraction nonconforming $p$ up to $15 p_{0}$ were displayed for a chosen design of the geometric CUSUM chart. The CCC-3 chart and the geometric CUSUM chart (and $\mathrm{np}$ chart) with the random-shift model were considered in Wu et al. (2000). Small in-control $p_{0}$ between 0.0001 and 0.00065 were assumed, the specified $p_{1}$ for the upward shift detection ranged from $p_{0}$ to $10 p_{0}$ and only $\operatorname{ANOS}\left(p_{1}\right)$ was tabulated. Chang and Gan (2001) compared the CCC chart and the geometric, Bernoulli and binomial CUSUM charts. The zero-state and the case $p_{0}=0.0001, p_{1}=0.0003$ were considered. The ANOS values were tabulated for $p$ up to 10,000 $p_{0}$. The $\mathrm{RL}_{2}$ (or CCC-2) chart and the geometric CUSUM (apart from the Poisson CUSUM chart and the optimal $\mathrm{np}$ chart with curtailment) were compared in (Wu, 2006). The steadystate analysis with the random-shift model was considered for $p_{0}$ ranging from 0.005 to 0.02 , $p_{1}$ equal to $3 p_{0}$ or $5 p_{0}$, and $A N O S_{0}$ equal to $10 / p_{0}$ and $100 / p_{0}$. More extensive comparisons of the moving sums $\mathrm{RL}_{2}$ and the geometric CUSUM (among others) can be found in Bourke (2008). Here both the initial-state and steady-state random-shift analysis were performed. The $p_{0}$ values were between 0.002 and 0.01 . For some specified values of $A N O S_{0}$ expressed as multiples of $1 / p_{0}$ and ranging between 1,000 and 50,000 the $A N O S\left(p_{1}\right)$ was given, $p_{1}$ values were between $3 p_{0}$ and $20 p_{0}$.

In most works the geometric CUSUM chart was considered to be the best method that is capable to detect an increase in $p$ more quickly than other charts, except for very large shifts, when the ANOS in the CCC-r charts was smaller. The conclusions in Wu et al. (2000) were rather different; the higher quickness of the SCRL (CCC-3) chart was sometimes manifested even for smaller shifts. The difference may be caused by the fact that both the downward and upward shifts were considered in the study. As the surprising finding concerned the value of $p_{0}$ similar to ours, the comparison with our results may be of interest.

\subsection{Estimation of ANOS for CCC-CUSUM Chart}

Three scenarios mentioned above were considered: zero state, fixed-shift steady state, and random-shift steady state. The shift of the fraction nonconforming was represented by the change from $p_{0}$ to $p$. The moment of the change depended on a scenario.

\section{a) Zero state}

The process shift occurred immediately. All observations came from the process with the fraction nonconforming $p$, i.e. all generated observations followed the geometric distribution $\mathrm{G}(p)$. 


\section{b) Steady state, fixed shift}

Sometimes the necessity of a large number of in-control observations is emphasized when the steady-state model is to be analyzed. For example, Szarka and Woodall (2012) use 5,000 observations from $\mathrm{G}\left(p_{0}\right)$ before a shift occurs. This requirement seems to be worthless with respect to the random in-control variation of the CUSUM statistic. Here another method was used. The number of observations from $\mathrm{G}\left(p_{0}\right)$ was much smaller and not fixed, but it was restricted by the limit of 50,000 Bernoulli observations. Geometric observations $Y_{i}$ from $\mathrm{G}\left(p_{0}\right)$ were generated and at the same time, their cumulative sum was recorded. As soon as the cumulative sum exceeded the predetermined value of 50,000 for some $i=I$, the $Y$, value was cancelled and the change point was set immediately after $I-1$ observations from $G\left(p_{0}\right)$. All subsequent observations were from $G(p)$.

\section{c) Steady state, random shift}

Now the change point was set after a fixed number of 50,000 inspected items, i.e. randomly between two subsequent observed nonconforming items. Again, observations from $\mathrm{G}\left(p_{0}\right)$ were generated. As soon as their cumulative sum exceeded the value of 50,000 for some $i=l$, only a part $Z_{\text {, of }}$ this $Y$, obtained as a difference between 50,000 and the prior cumulative sum was taken into account. Then instead of $Y_{1}$ the sum $Z_{I}+Y_{i+1}$ was considered, where $Y_{i+1}$ came from $\mathrm{G}(p)$. All subsequent observations were from $\mathrm{G}(p)$.

In both cases b) and c) false signals before the process shift were excluded; when the CUSUM statistic exceeded the lower limit $H$, the current observation was cancelled and another one was generated.

\subsection{Calculating ANOS for CCC-r Chart}

The values of ANOS for the CCC-r charts were determined according to the formula

$$
A N O S=\frac{r}{p \cdot F(L C L)}
$$

where $F(L C L)$ can be set using equation (9).

The theoretical value $A N O S_{0}$ for $p=p_{0}$ is

$$
A N O S_{0}=\frac{r}{p_{0} \cdot \alpha}
$$

In the case of the steady-state randomshift model, ANOS for CCC-r was estimated using simulation as follows: the run lengths $Y_{i}$ from $\mathrm{G}\left(p_{0}\right)$ and $\mathrm{G}(p)$ were generated as in $c$ ) of the previous section 2.1 but now sums of three (or two) subsequent observations were calculated and after each new triple (pair) the sum was compared with the corresponding lower control limit $L C L$. The cumulative sum of run lengths $N^{\prime} S_{i}$ was calculated as before and as soon as the current sum of three (two) run lengths decreased under $L C L$ for some $i=I$, the simulation was interrupted and $N O S_{i}$ was stored. To arrange the same sequence of observations as in 2c), the part of the former procedure for the geometric CUSUM with cancelling observations that led to false alarms was used.

\section{Comparative Statistical Analysis}

Based on the experience with the manufacturing process which is described in detail in Section 4, the in-control $p_{0}=0.0002$ and the out-of-control $p_{1}=5 p_{0}$ were chosen. Three values of the risk $\alpha$ were considered: $0.0027,0.005$, and 0.01 . The semi-economic model by Brodecká (2013) resulted in the CCC-3 chart for $\alpha=0.0027$ and the CCC-2 chart for $\alpha$ equal to 0.005 or 0.01 . The lower limits for both types are displayed in Tab. 1.

The fraction nonconforming $p$ ranged from 0.0004 to 0.003 . The values of ANOS for all charts are shown in Tab. 2, 3 and 4 and in Fig. 1, 2 and 3.

\section{Tab. 1: Lower limits of CCC-r chart and geometric CUSUM chart}

\begin{tabular}{c|c|c|r|rr} 
& & \multicolumn{2}{|c|}{ CCC-r } & \multicolumn{2}{|c}{ Geometric CUSUM } \\
\hline $\boldsymbol{\alpha}$ & $\boldsymbol{r}$ & $\boldsymbol{L C L}$ & $\boldsymbol{H}_{\boldsymbol{G}}$ & $\boldsymbol{H}$ \\
\hline 0.0027 & 3 & 1,354 & $-7,171$ & $-7,179$ \\
\hline 0.0050 & 2 & 518 & $-5,901$ & $-5,904$ \\
\hline 0.0100 & 2 & 744 & $-5,044$ & $-5,061$ \\
\hline
\end{tabular}




\section{Business Administration and Management}

Tab. 2:

ANOS for the geometric CUSUM (three models of the shift occurrence)

and the CCC-3 chart (formula (14) and simulation), $\alpha=0.0027$

\begin{tabular}{c|r|r|r|r|r} 
& \multicolumn{4}{|c|}{ CUSUM } & \multicolumn{2}{c}{ CCC-3 } \\
\hline $\boldsymbol{p}$ & \multicolumn{1}{|c|}{ zero } & \multicolumn{1}{c}{ fixed } & \multicolumn{1}{c}{ random } & traditional & \multicolumn{1}{c}{ random } \\
\hline 0.0002 & $5,557,857$ & $5,557,857$ & $5,557,857$ & $5,566,358$ & $5,557,857$ \\
\hline 0.0004 & 116,645 & 115,474 & 117,576 & 424,203 & 245,093 \\
\hline 0.0006 & 24,954 & 24,727 & 25,981 & 101,870 & 64,965 \\
\hline 0.0008 & 11,867 & 11,675 & 12,637 & 39,066 & 26,577 \\
\hline 0.0010 & 7,624 & 7,482 & 8,204 & 19,332 & 14,328 \\
\hline 0.0012 & 5,561 & 5,446 & 6,076 & 11,226 & 9,088 \\
\hline 0.0014 & 4,390 & 4,286 & 4,837 & 7,271 & 6,379 \\
\hline 0.0016 & 3,613 & 3,519 & 3,994 & 5,097 & 4,885 \\
\hline 0.0018 & 3,075 & 2,996 & 3,419 & 3,791 & 3,904 \\
\hline 0.0020 & 2,674 & 2,606 & 2,979 & 2,952 & 3,201 \\
\hline 0.0022 & 2,366 & 2,308 & 2,643 & 2,385 & 2,726 \\
\hline 0.0024 & 2,123 & 2,073 & 2,365 & 1,984 & 2,370 \\
\hline 0.0026 & 1,923 & 1,873 & 2,146 & 1,690 & 2,124 \\
\hline 0.0028 & 1,757 & 1,714 & 1,972 & 1,468 & 1,912 \\
\hline 0.0030 & 1,618 & 1,580 & 1,820 & 1,297 & 1,743 \\
\hline
\end{tabular}

Source: own

Tab. 3: ANOS for the geometric CUSUM (three models of the shift occurrence) and the CCC-2 chart (formula (14) and simulation), $\alpha=0.005$

\begin{tabular}{c|r|r|r|r|r} 
& \multicolumn{4}{|c|}{ CUSUM } & \multicolumn{2}{c}{ CCC-2 } \\
\hline $\mathbf{p}$ & \multicolumn{1}{|c|}{ zero } & \multicolumn{1}{c|}{ fixed } & \multicolumn{1}{c}{ random } & \multicolumn{1}{c}{ traditional } & \multicolumn{1}{c}{ random } \\
\hline 0.0002 & $1,999,842$ & 1,999842 & $1,999,842$ & $2,006,896$ & $1,999,842$ \\
\hline 0.0004 & 77,281 & 77,227 & 79,117 & 268,458 & 273,083 \\
\hline 0.0006 & 19,729 & 19,481 & 20,728 & 85,071 & 86,770 \\
\hline 0.0008 & 9,850 & 9,707 & 10,639 & 38,361 & 40,558 \\
\hline 0.0010 & 6,372 & 6,197 & 6,996 & 20,980 & 22,525 \\
\hline 0.0012 & 4,694 & 4,554 & 5,197 & 12,961 & 14,153 \\
\hline 0.0014 & 3,702 & 3,594 & 4,133 & 8,708 & 9,781 \\
\hline 0.0016 & 3,053 & 2,972 & 3,442 & 6,220 & 7,189 \\
\hline 0.0018 & 2,595 & 2,538 & 2,940 & 4,655 & 5,480 \\
\hline 0.0020 & 2,260 & 2,208 & 2,572 & 3,613 & 4,308 \\
\hline 0.0022 & 2,003 & 1,955 & 2,282 & 2,889 & 3,527 \\
\hline 0.0024 & 1,798 & 1,756 & 2,059 & 2,366 & 2,971 \\
\hline 0.0026 & 1,639 & 1,596 & 1,881 & 1,978 & 2,552 \\
\hline 0.0028 & 1,503 & 1,460 & 1,725 & 1,682 & 2,216 \\
\hline 0.0030 & 1,388 & 1,348 & 1,596 & 1,451 & 1,933 \\
\hline
\end{tabular}


Tab. 4: ANOS for the geometric CUSUM (three models of the shift occurrence) and the CCC-2 chart (formula (14) and simulation), $\alpha=0.01$

\begin{tabular}{c|r|r|r|r|r} 
& \multicolumn{4}{|c|}{ CUSUM } & \multicolumn{2}{c}{ CCC-2 } \\
\hline $\mathbf{p}$ & \multicolumn{1}{|c|}{ zero } & \multicolumn{1}{c|}{ fixed } & \multicolumn{1}{c}{ random } & traditional & \multicolumn{1}{c}{ random } \\
\hline 0.0002 & $1,000,268$ & $1,000,268$ & $1,000,268$ & $1,000,511$ & $1,000,268$ \\
\hline 0.0004 & 57,341 & 57,770 & 59,423 & 137,798 & 140,892 \\
\hline 0.0006 & 16,510 & 16,264 & 17,619 & 44,930 & 47,776 \\
\hline 0.0008 & 8,482 & 8,280 & 9,270 & 20,832 & 22,720 \\
\hline 0.0010 & 5,558 & 5,425 & 6,190 & 11,707 & 13,244 \\
\hline 0.0012 & 4,092 & 3,987 & 4,608 & 7,426 & 8,661 \\
\hline 0.0014 & 3,223 & 3,148 & 3,676 & 5,120 & 6,101 \\
\hline 0.0016 & 2,662 & 2,599 & 3,050 & 3,750 & 4,632 \\
\hline 0.0018 & 2,249 & 2,202 & 2,625 & 2,875 & 3,699 \\
\hline 0.0020 & 1,958 & 1,919 & 2,297 & 2,285 & 3,028 \\
\hline 0.0022 & 1,728 & 1,694 & 2,039 & 1,869 & 2,527 \\
\hline 0.0024 & 1,545 & 1,508 & 1,830 & 1,565 & 2,157 \\
\hline 0.0026 & 1,394 & 1,363 & 1,665 & 1,336 & 1,894 \\
\hline 0.0028 & 1,269 & 1,242 & 1,524 & 1,160 & 1,676 \\
\hline 0.0030 & 1,168 & 1,142 & 1,406 & 1,021 & 1,504 \\
\hline & & & & & Source: own
\end{tabular}

\section{Fig. 1: ANOS curves for the CCC-3 and the geometric CUSUM chart, $\alpha=0.0027$}

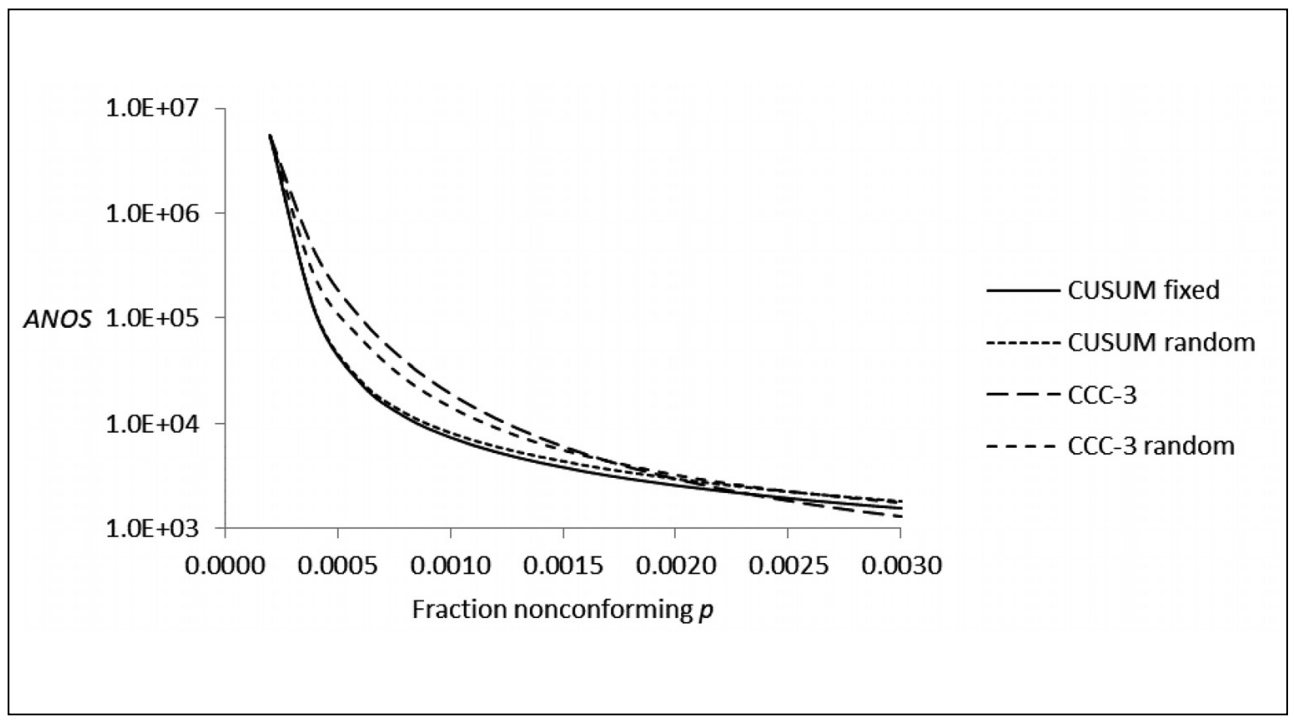




\section{Business Administration and Management}

Fig. 2: ANOS curves for the CCC-2 and the geometric CUSUM chart, $\alpha=0.005$

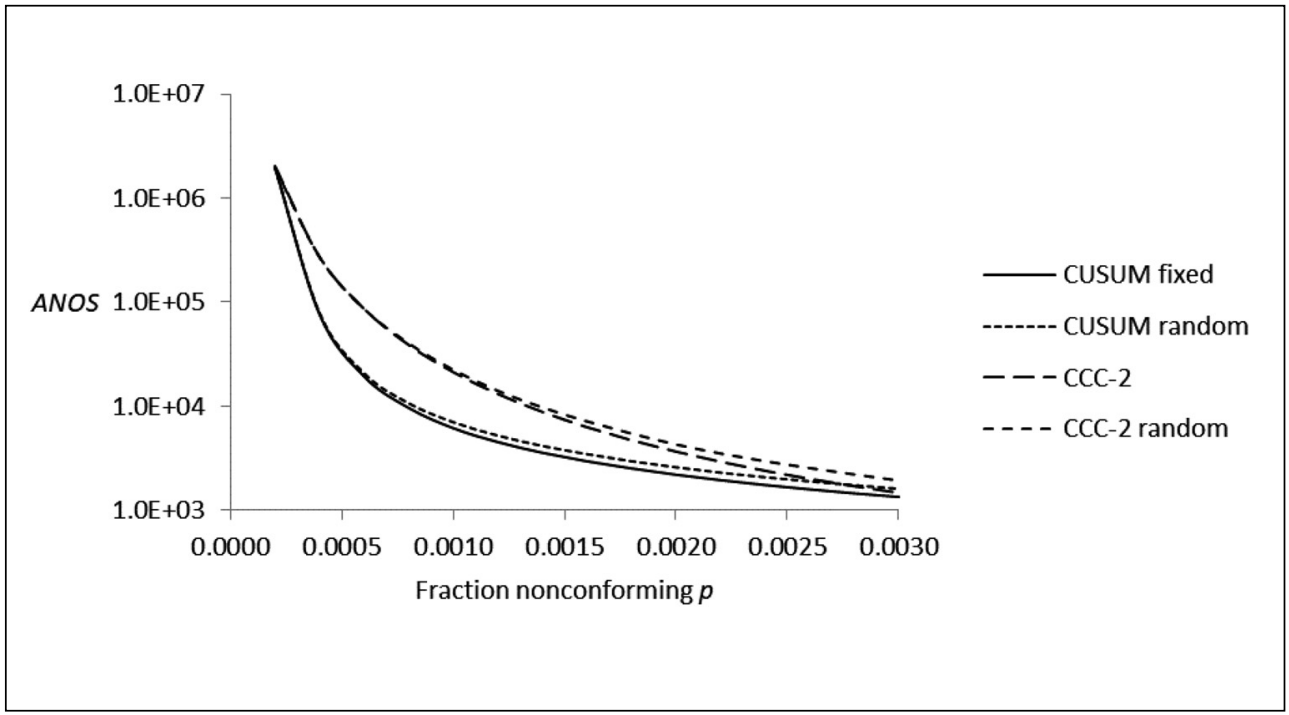

Source: own

\section{Fig. 3:}

ANOS curves for the CCC-2 and the geometric CUSUM chart, $\alpha=0.01$

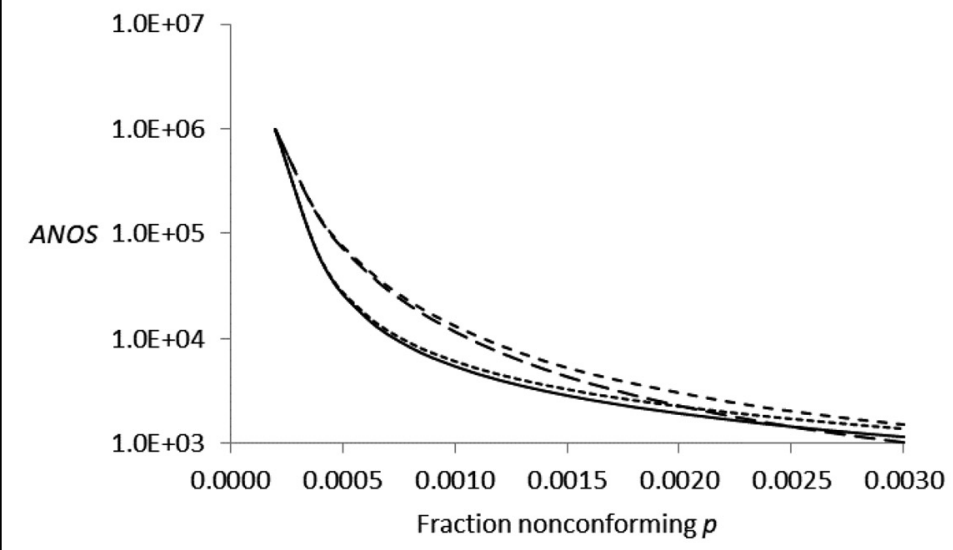

- CUSUM fixed

CUSUM random

$--\mathrm{CCC}-2$

- - - - CCC-2 random 
As for the geometric CUSUM chart, ANOS for the steady-state fixed-shift model is smaller than for the zero state as could be expected, because on average, the CUSUM statistic is less than zero and nearer to the lower limit $H$ then. Obviously, the differences between the two models are negligible and so only the fixedshift and random-shift models are used in the subsequent analysis. ANOS for the randomshift model is always larger than that for the fixed-shift model, which follows from the fact that the run length covering the change point tends to be larger than the run length starting from the change point.

The CCC-2 chart shows the similar performance as to the differences between the fixed-shift and random-shift models. With the CCC-3 chart, the relation between ANOS for the random-shift model and that for the fixedshift model is reverse for $p$ up to 0.0016 .

To compare the CCC-r and geometric CUSUM charts, the efficiency ratio

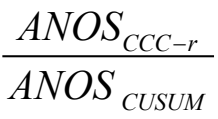

was used. Both the fixed- and random-shift models were considered (Tab. 5). They confirm that in terms of ANOS, the geometric CUSUM chart performs better than the corresponding CCC-r chart for $p$ up to 0.0022 , i.e. approximately up to more than $2 p_{1}$. Larger shifts may be detected more quickly by CCC-r chart, depending on $A N O S_{0}$ which is related to the risk $\alpha$, and the type of the shift considered.

The results are consistent with those discussed in the literature sources mentioned above. The superiority of the CCC-r chart over the geometric CUSUM chart for small shifts and small $p_{0}$ claimed in Wu et al. (2000) was not confirmed.

\section{Tab. 5: Efficiency ratio ANOS ccc-r $_{\text {/ }}$ ANOS $_{\text {cusum }}$}

\begin{tabular}{|c|c|c|c|c|c|c|}
\hline \multirow{2}{*}{$p$} & \multicolumn{2}{|c|}{$\begin{array}{c}\alpha=0.0027 \\
r=3\end{array}$} & \multicolumn{2}{|c|}{$\begin{array}{c}\alpha=0.005 \\
r=2\end{array}$} & \multicolumn{2}{|c|}{$\begin{array}{c}\alpha=0.01 \\
r=2\end{array}$} \\
\hline & fixed & random & fixed & random & fixed & random \\
\hline 0.0002 & 1.002 & 1.000 & 1.004 & 1.000 & 1.000 & 1.000 \\
\hline 0.0004 & 3.674 & 2.085 & 3.476 & 3.452 & 2.385 & 2.371 \\
\hline 0.0006 & 4.120 & 2.500 & 4.367 & 4.186 & 2.763 & 2.712 \\
\hline 0.0008 & 3.346 & 2.103 & 3.952 & 3.812 & 2.516 & 2.451 \\
\hline 0.0010 & 2.584 & 1.747 & 3.385 & 3.220 & 2.158 & 2.140 \\
\hline 0.0012 & 2.061 & 1.496 & 2.846 & 2.723 & 1.863 & 1.880 \\
\hline 0.0014 & 1.697 & 1.319 & 2.423 & 2.367 & 1.626 & 1.660 \\
\hline 0.0016 & 1.448 & 1.223 & 2.093 & 2.089 & 1.443 & 1.519 \\
\hline 0.0018 & 1.266 & 1.142 & 1.834 & 1.864 & 1.306 & 1.409 \\
\hline 0.0020 & 1.133 & 1.075 & 1.637 & 1.675 & 1.191 & 1.318 \\
\hline 0.0022 & 1.033 & 1.032 & 1.478 & 1.545 & 1.103 & 1.239 \\
\hline 0.0024 & 0.957 & 1.002 & 1.348 & 1.443 & 1.038 & 1.178 \\
\hline 0.0026 & 0.902 & 0.990 & 1.240 & 1.357 & 0.981 & 1.137 \\
\hline 0.0028 & 0.857 & 0.970 & 1.152 & 1.284 & 0.934 & 1.100 \\
\hline 0.0030 & 0.821 & 0.958 & 1.076 & 1.212 & 0.894 & 1.070 \\
\hline
\end{tabular}




\section{Application to the Assembling Process}

CCC-r and CCC-CUSUM charts were applied to the highly capable process of the Exhaust Gas Recirculation (EGR) pressure sensor manufacturing. This sensor detects the exhaust gas flow in the EGR valve which is a part of the combustion motor that enables to reduce nitrogen oxide (NOx) emissions. The electronic assembling of the EGR sensor consists of several subprocesses and operations. The last but one subprocess is the manual application of the protective gel. This subprocess is highly capable with in-control fraction nonconforming $p_{0}=0.0002(200 \mathrm{ppm})$. Every sensor is visually inspected - it means that continuous 100\% inspection is performed. This subprocess was chosen for the application of SPC based on the Bernoulli process to obtain information about changes in the fraction nonconforming. The problem of controlling this process is solved in Brodecka (2013). She considered CCC-r chart and proposed a new semi-economic model which is based on the economic design of CCC-r chart described in Ohta et al. (2001) but is easier to apply in practice. The algorithm of the new semi-economic model is based on the minimization of the overall cost of producting nonconforming units, inspection and testing, and parameter $r$ is the only optimized parameter.

Three values of the risk of false signal $\alpha$ were considered in our application and therefore three control charts for each type were constructed. For $\alpha=0.0027$, the parameter $r=3$ was set as the optimal one using the semi-economic model. Separated sums of three observed successive run lengths are plotted in Fig. 4. The lower limit of 1,354 was not exceeded.

For $\alpha=0.005$ and $\alpha=0.01$ the parameter $r=2$ was set as the optimal one and the CCC-2 chart with the limits of 518 and 744 , respectively, was applied. Now, the sums of two successive run lengths were plotted (Fig. 5). The lower limit $L C L$ was not exceeded in any of the cases.

Fig. 6 shows the geometric CUSUM chart. Three variants of the lower limit $H(-7,179 ;-5,904$; $-5,061)$ for three values of $\alpha$ are displayed. For $\alpha=0.01$, the signal occurred at the $50^{\text {th }}$ observed run length, in the case of $\alpha=0.005$ at the $51^{\text {st }}$ observation.

The results obtained correspond to the analysis described in Section 3. An upward shift in fraction nonconforming was likely to occur sometime after the $45^{\text {th }}$ nonconforming unit was found. However, the shift was not large and therefore the $16^{\text {th }}$ and $17^{\text {th }}$ observation in

\section{Fig. 4: CCC-3 chart for the gel-application process control}

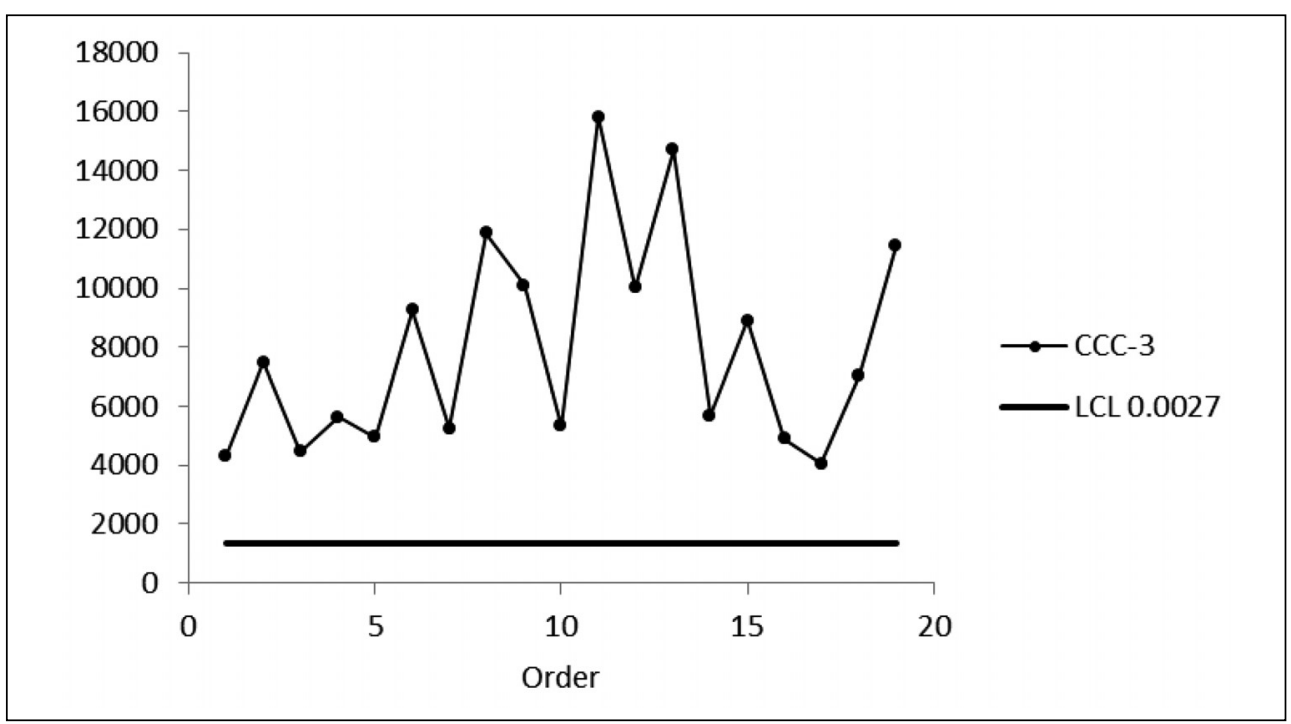


Fig. 5: CCC-2 chart for the gel-application process control

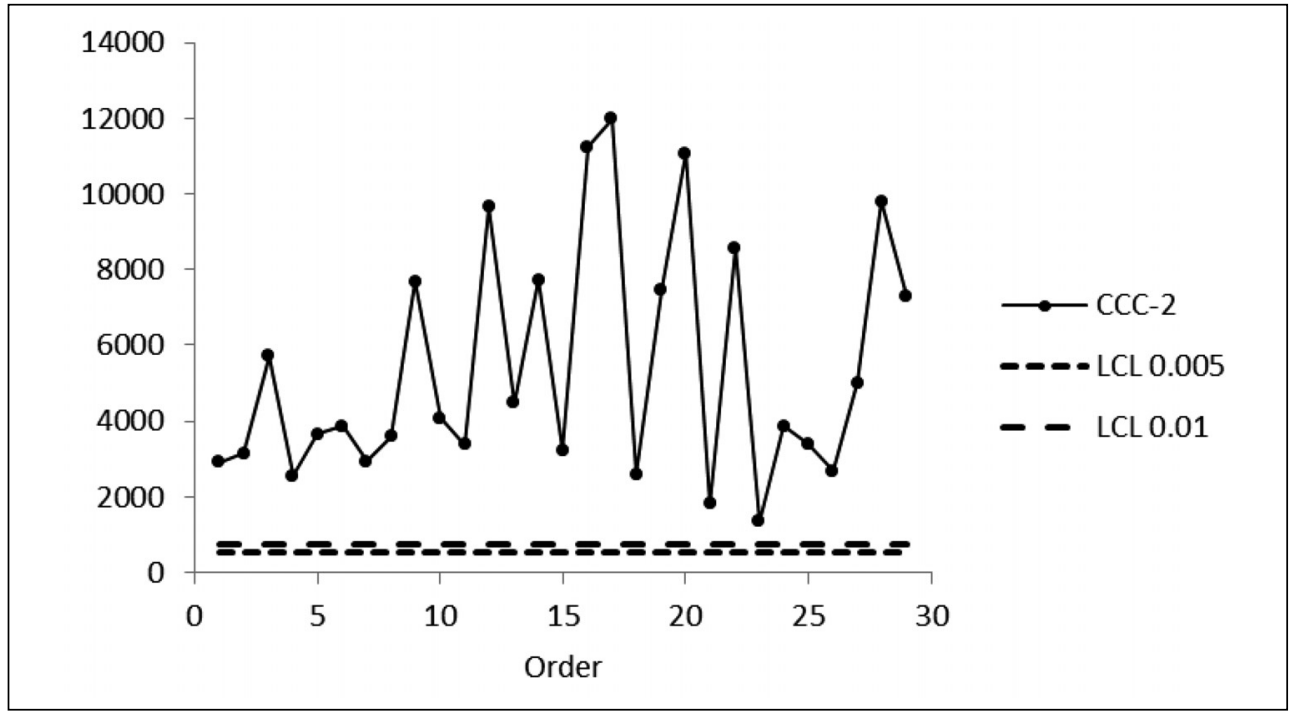

Source: own

Fig. 6: CCC-CUSUM chart for the gel-application process control

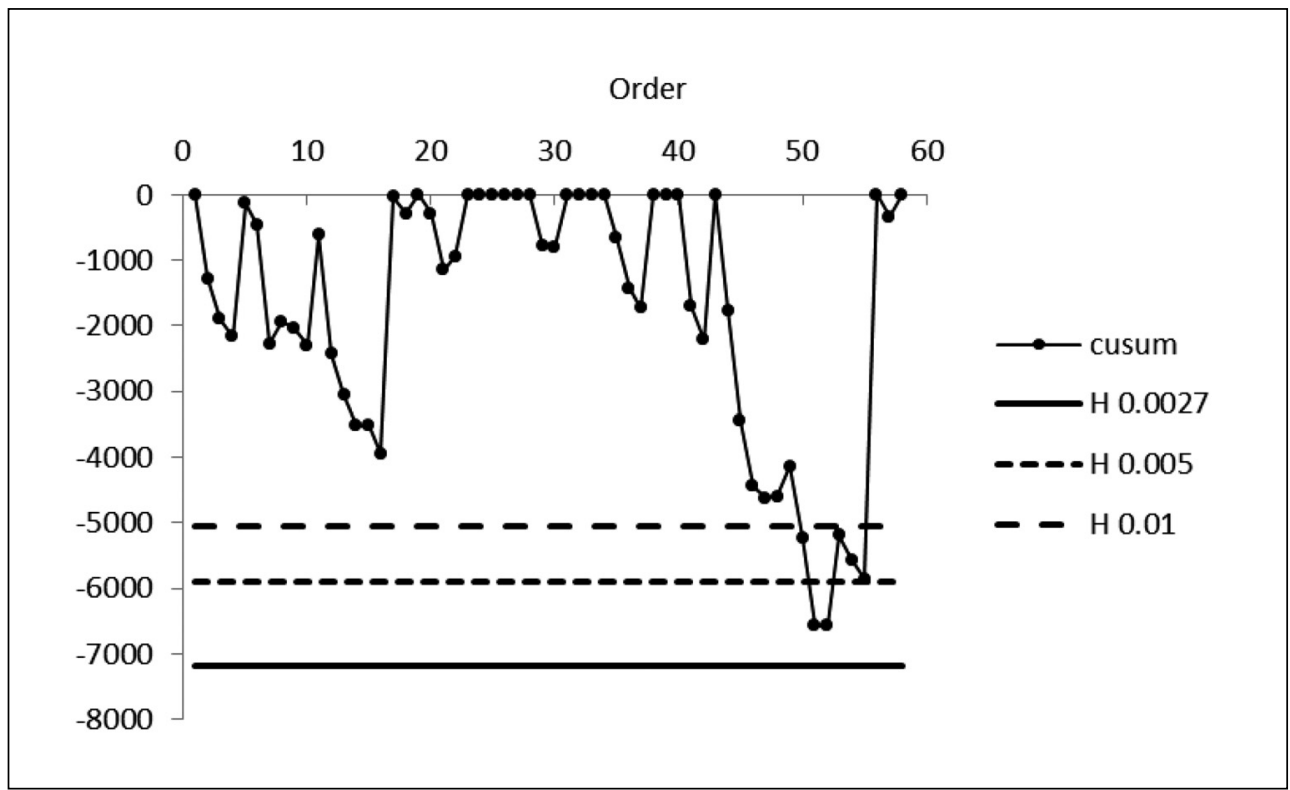


Fig. 4 referring to the $46^{\text {th }}$ to $51^{\text {st }}$ nonconforming units, or the $23^{\text {rd }}$ observation in Fig. 5 referring to the $45^{\text {th }}$ and $46^{\text {th }}$ nonconforming units only approached the lower limit. The CUSUM chart for higher values of $\alpha$ responded to this shift after the occurrence of several other nonconforming units.

\section{Conclusions}

In this paper, the efficiency of two types of CCC charts was studied using the expected number of inspected items to the signal ANOS. Two scenarios of upward shifts were considered: the fixed-shift and random-shift model. The latter model is considered more realistic, but the differences in ANOS are not too great, especially for $\alpha$ of 0.005 or 0.01 .

Since the response of the CUSUM chart to an upward shift in fraction nonconforming is much faster than that of the CCC-r chart for shifts up to ten times the in-control fraction nonconforming, the superiority of the geometric CUSUM was confirmed. For example, considering the random-shift model, $\alpha=0.005$ (or ANOS $_{0}$ around 2,000,000) and the shift from $p_{0}=0.0002$ to $p_{1}=0.001$, for which the CUSUM chart was designed, the signal can be expected after approximately 7 nonconforming units on average compared with 23 nonconforming units with the CCC-2 chart that must be discovered before the signal occurs. In this case, the production of another 16 nonconforming units may be prevented, representing approximately $70 \%$ saving cost for repair or replacement of nonconforming units.

The response to larger shifts is comparable for both types of charts. Therefore, when larger shifts are to be avoided, the CCC-r chart may be preferred due to its easier construction.

This paper was elaborated in the frame of the specific research project SP2018/109 and SP2019/62, which has been solved at the Faculty of Materials Science and Technology, VŠB - TU Ostrava with the support of Ministry of Education, Youth and Sports, Czech Republic, and the Skoda Auto University project.

\section{References}

Bourke, P. D. (1991). Detecting a Shift in Fraction Nonconforming using Run-Length Control Charts with $100 \%$ Inspection. Journal of Quality Technology, 23(13), 225-238. https://doi.org/10.1080/00224065.1991.11979328.
Bourke, P. D. (2001). The Geometric CUSUM chart with Sampling Inspection for Monitoring Fraction Defective. Journal of Applied Statistics, 28(8), 951-972. https://doi. org/10.1080/02664760120076643.

Bourke, P. D. (2006). The $\mathrm{RL}_{2}$ Chart versus the $\mathrm{np}$ Chart for Detecting Upward Shifts in Fraction Defective. Journal of Applied Statistics, 33(1), 1-15. https://doi.org/10.1080/02664760500389400.

Bourke, P. D. (2008). Performance Comparisons for the Synthetic Control Chart for Detecting Increases in Fraction Nonconforming. Journal of Quality Technology, 40(4), 461-475. https://doi.org/10.1080/00224065.2008.11917749.

Brodecká, K. (2013). Application of Selected Statistical Methods at Conditions of High Yield Processes (in Czech). Doctoral Thesis, VŠBTU Ostrava, Ostrava.

Brook, D., \& Evans, D. A. (1972). An Approach to the Probability Distribution of Cusum Run Length. Biometrika, 59(3), 539-549. https://doi.org/10.1093/biomet/59.3.539.

Calvin, T. (1983). Quality Control Techniques for Zero Defects. IEEE Transactions on Components Hybrids and Manufacturing Technology, 6(3), 323-328. https://doi. org/10.1109/TCHMT.1983.1136174.

Chan, L. Y., Xie, M., \& Goh, T. N. (2000). Cumulative Quantity Control Charts for Monitoring Production Processes. International Journal of Production Research, 38(2), 399-408. https://doi.org/10.1080/002075400189482.

Chang, T. C., \& Gan, F. F. (2001). Cumulative Sum Charts for High Yield Processes. Statistica Sinica, 11(3), 791-805.

Chang, T. C., \& Gan, F. F. (2007). Modified Shewhart Charts for High Yield Processes. Journal of Applied Statistics, 34(7), 857-877. https://doi.org/10.1080/02664760701546279.

Chen, P. W., \& Cheng, C. S. (2010). An ARL-Unbiased Approach to Setting Control Limits of CCC-r Chart for High Yield Processes. Journal of Quality, 17(6), 435-451. https://doi. org/10.6220/joq.

Di Bucchianico, A., Mooiweer, G. D., \& Moonen, E. J. G. (2005). Monitoring Infrequent Failures of High-volume Production Processes. Quality and Reliability Engineering International, 21(5), 521-528. https://doi.org/10.1002/qre.738.

Goh, T. N. (1987). A Control Chart for Very High Yield Processes. Quality Assurance, 13(1), 18-22.

Hawkins, D. M., \& Olwell, D. H. (1998). Cumulative Sum Charts and Charting for Quality Improvement. New York: Springer. 
Kaminsky, F. C., Benneyan, J. C., Davis, R. D., \& Burke, R. J. (1992). Statistical Control Charts Based on a Geometric Distribution. Journal of Quality Technology, 24(2), 63-69. https://doi.org/10.1080/00224065.1992.12015 229.

Montgomery, D. C. (2009). Statistical Quality Control: A Modern Introduction. Hoboken: J. Wiley \& Sons.

Ohta, H., Kusukawa, E., \& Rahim, A. (2001). A CCC-r Chart for High-Yield Processes. Quality and Reliability Engineering International, 17(6), 439-446. https://doi.org/10.1002/qre.428.

Page, E. S. (1954). Continuous Inspection Schemes. Biometrika, 41(1), 100-114. https://doi.org/10.1093/biomet/41.1-2.100.

Reynolds, M. R., \& Stoumbos, Z. G. (1999). A CUSUM Chart for Monitoring a Proportion When Inspecting Continuously. Journal of Quality Technology, 31(1), 87-108. https://doi. org/10.1080/00224065.1999.11979900.

Schwertman, N. C. (2005). Designing Accurate Control Charts Based on the Geometric and Negative Binomial Distribution. Quality and Reliability Engineering International, 21(8), 743-756. https://doi.org/10.1002/qre.683.

Szarka, J. L. III, \& Woodall, W. H. (2011). A Review and Perspective on Surveillance of Bernoulli Processes. Quality and Reliability Engineering International, 27(6), 735-752. https://doi.org/10.1002/qre.1256.

Szarka, J. L. III, \& Woodall, W. H. (2012). On the Equivalence of the Bernoulli and Geometric CUSUM Charts. Journal of Quality Technology, 44(1), 54-62. https://doi.org/10.1080/00224065. 2012.11917881.

Wu, Z., \& Spedding, T. A. (1999). Evaluation of ATS for CRL Control Chart. Process Control and Quality, 11(3), 183-191. https://doi. org/10.1163/156856699750248540.

Wu, Z., Yeo, S. H., \& Fan, H. (2000). A Comparative Study of the CRL-Type Control Charts. Quality and Reliability Engineering International, 16(4), 269-279. https://doi. org/10.1002/1099-1638(200007/08)16:4<269::AIDQRE334>3.0.CO;2-D.

Wu, Z., Zhang, X., \& Yeo, S. H. (2001). Design of the Sum-of-Conforming-RunLength Control Charts. European Journal of Operational Research, 132(1), 187-196. https://doi.org/10.1016/S0377-2217(00)00131-4.
Wu, Z., Luo, H., \& Zhang, X. L. (2006). Optimal np Control Chart with Curtailment. European Journal of Operational Research, 174(3), 1723-1741. https://doi.org/10.1016/j. ejor.2005.03.038.

Xie, W., Xie, M., \& Goh, T. N. (1995). A Shewhart-like Charting Technique for High Yield Processes. Quality and Reliability Engineering International, 11(3), 189-196. https://doi.org/10.1002/qre.4680110309.

Xie, M., \& Goh, T. N. (1997). The Use of Probability Limits for Process Control Based on Geometric Distribution. International Journal of Quality and Reliability Management, 14(1), 64-73. https://doi. org/10.1108/02656719710156789.

Xie, M., Lu, X. S., Goh, T. N., \& Chan, L. Y. (1999). A Quality Monitoring and Decisionmaking Scheme for Automated Production Processes. International Journal of Quality and Reliability Management, 16(2), 148-157. https:// doi.org/10.1108/02656719910218238.

Xie, M., Goh, T. N., \& Kuralmani, C. (2002). Statistical Models and Control Charts for High Quality Processes. Norwel, Massachusetts: Kluwer Academic Publishers.

Yeh, A. B., McGrath, R. N., Sembower, M. A., \& Shen, Q. (2008). EWMA Control Charts for Monitoring High-yield Processes Based on Nontransformed Observations. International Journal of Production Research, 46(20), 5679-5699. https://doi.org/10.1080/00207540601182252.

Zhang, L., Govindaraju, K., Bebbington, M., \& Lai, C. D. (2004). On the Statistical Design of Geometric Control Charts. Quality Technology and Quantitative Management, 1(2), 233-243. https://doi.org/10.1080/16843703.2004.11673075.

doc. Ing. Eva Jarošová, CSc.

Skoda Auto University Department of Logistics and Quality Management Czech Republic jarosova@is.savs.cz

prof. Ing. Darja Noskievičová, CSc.

VSB - Technical University of Ostrava Faculty of Materials Science and Technology Department of Quality Management Czech Republic darja.noskievicova@vsb.cz 


\section{Abstract}

\section{COMPARATIVE STATISTICAL ANALYSIS OF SELECTED CONTROL CHARTS FOR HIGHLY CAPABLE PROCESSES}

\section{Eva Jarošová, Darja Noskievičová}

When a high-quality process is to be controlled by $100 \%$ inspection and yes-no decision is employed, several types of charts come into account, e.g. CCC, CCC-r or geometric CUSUM (CCC-CUSUM). The aim of the paper is to examine performance of these charts so that a suitable one can be chosen for a given process. The charts are compared according to the quickness with which the upward shift in the fraction of nonconforming items is detected. The average number of observations to signal (ANOS) instead of the usual average run length (ARL) is determined. While ANOS for CCC or CCC-r charts can be easily calculated based on a geometric or a negative binomial distribution, its computation is quite difficult in the case of CCC-CUSUM chart. The corrected diffusion (CD) approximation was used to determine ANOS and the results were verified by Monte Carlo simulation. Zero-state and steady-state (both fixed-shift and random-shift model) analyses were performed to take different scenarios of the process shift occurrence into account. CCC-3 or CCC-2 and CCC-CUSUM charts were compared. The order $r$ for CCC-r chart was chosen as an optimal value for the given process based on the semi-economic model suggested in Brodecká (2013). Our study revealed that for in-control $p_{0}$ equal to 0.0002 the CCC-CUSUM chart performs best especially for shifts around the pre-specified out-of-control fraction nonconforming. The CCC-r chart may be comparable or even better in detecting larger shifts. The results of the comparative study were utilized for the choice of the most suitable and best performing control chart to control the high-yield process producing ERG (Exhaust Gas Recirculation) sensors. Comparisons of CCC-r and CCC-CUSUM charts can be found elsewhere in literature, but conclusions seem to be rather inconsistent. To our best knowledge no study dealing with such small in-control fraction nonconforming together with the low risk of false alarm has been published yet. The choice of CUSUM's parameters and consequent values of ANOS can help practitioners who need to control high-quality processes.

Key Words: CCC chart, CCC-r chart, CCC-CUSUM chart, ANOS, zero-state scenario, fixed shift steady-state scenario, random shift steady-state scenario, simulation.

JEL Classification: C46, L62.

DOI: 10.15240/tul/001/2019-2-005 